\title{
Turn-by-Wire: Computationally Mediated Physical Fabrication
}

\author{
Rundong Tian ${ }^{1,2}$, Vedant Saran ${ }^{1}$, Mareike Kritzler $^{2}$, Florian Michahelles ${ }^{2}$, Eric Paulos ${ }^{1}$ \\ ${ }^{1}$ Electrical Engineering and Computer Sciences \\ University of California, Berkeley \\ \{rutian, vedantsaran, paulos\}@ berkeley.edu
${ }^{2}$ Artificial \& Human Intelligence Research Siemens Corporate Technology, Berkeley, CA \{firstname.lastname\}@siemens.com

\begin{abstract}
Advances in digital fabrication have simultaneously created new capabilities while reinforcing outdated workflows that constrain how, and by whom, these fabrication tools are used. In this paper, we investigate how a new class of hybridcontrolled machines can collaborate with novice and expert users alike to yield a more lucid making experience. We demonstrate these ideas through our system, Turn-by-Wire. By combining the capabilities of a traditional lathe with haptic input controllers that modulate both position and force, we detail a series of novel interaction metaphors that invite a more fluid making process spanning digital, model-centric, computer control, and embodied, adaptive, human control. We evaluate our system through a user study and discuss how these concepts generalize to other fabrication tools.
\end{abstract}

\section{CCS Concepts}

-Human-centered computing $\rightarrow$ Human computer interaction (HCI); Haptic devices;

\section{Author Keywords}

Digital Fabrication; Augmented Tools; Haptics; Digital

Companions; Interactive Fabrication

\section{INTRODUCTION}

The proliferation of digital fabrication tools has empowered many makers with the ability to make nearly anything. Simultaneously however, the workflows surrounding these digital tools have narrowed the ways in which we interact with and conceptualize fabrication, steering a process as hands-on as "making" towards heavily computer-oriented workflows. Digital fabrication tools have their benefits: no prerequisite manual dexterity needs to be developed, extreme accuracy is achievable for highly complex geometries, and structured workflows are strictly enforced, to highlight a few.

While useful, we claim that these qualities of digital fabrication are not valued by all makers at all times. In some

Permission to make digital or hard copies of all or part of this work for personal or classroom use is granted without fee provided that copies are not made or distributed for profit or commercial advantage and that copies bear this notice and the full citation on the first page. Copyrights for components of this work owned by others than ACM must be honored. Abstracting with credit is permitted. To copy otherwise, or republish to post on servers or to redistribute to lists, requires prior specific permission and/or a fee. Request permissions from permissions@acm.org.

UIST'19, October 20-23, 2019, New Orleans, LA, USA.

Copyright is held by the owner/author(s). Publication rights licensed to ACM.

ACM ISBN 978-1-4503-6816-2/19/10 _.\$15.00.

http://dx.doi.org/10.1145/3332165.3347918
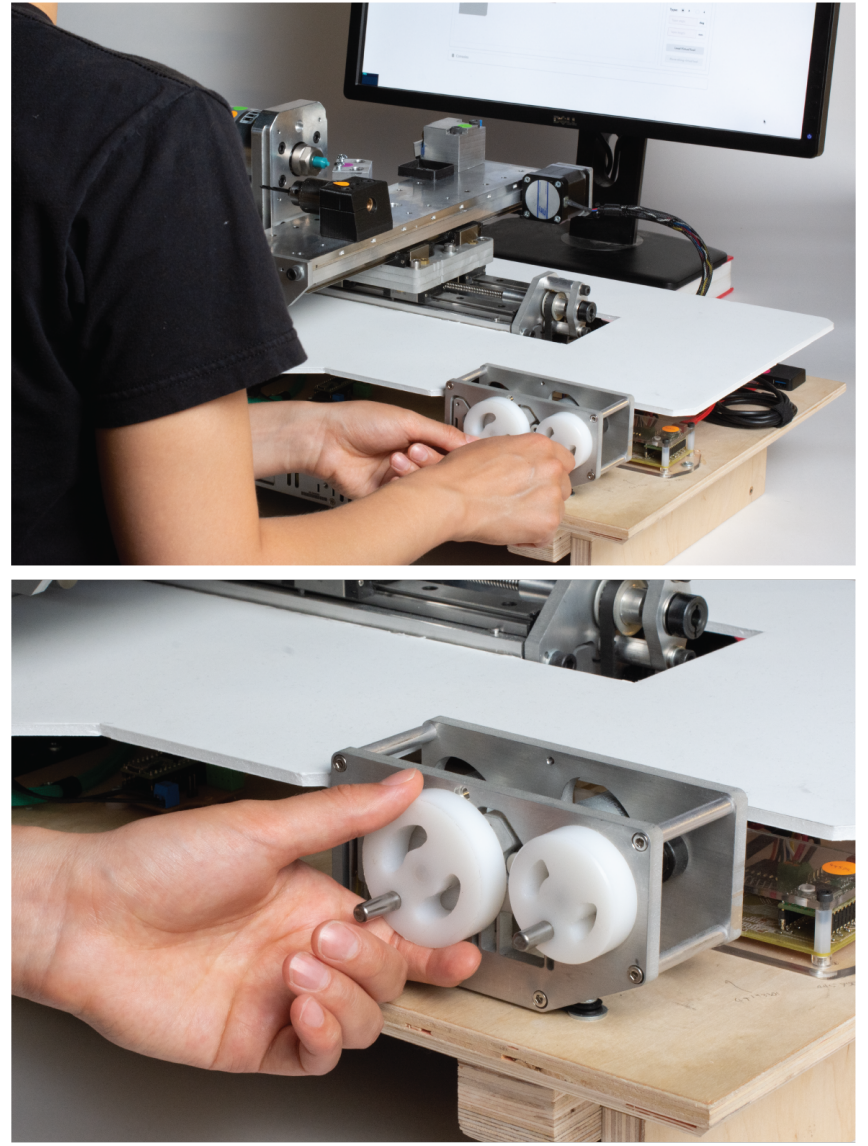

Figure 1. Top: the Turn-By-Wire system consists of a lathe, haptic feedback handwheels, and a graphical user interface. Bottom: detailed view of the handwheels used to directly and digitally control the lathe.

situations, other questions carry more weight: How might a skilled artisan incorporate their embodied knowledge within digital fabrication? How might we interact with fabrication tools differently if the maximum attainable precision and complexity is not always required? How might these fabrication tools recognize that design constraints are often flexible and ambiguous, and be similarly flexible in their workflows?

Researchers in HCI and beyond have approached these questions from a variety of perspectives: augmenting hand-held tools with sensing and actuation [36, 52, 38, 49], utilizing direct manipulation metaphors $[48,25]$, implementing bidirec- 


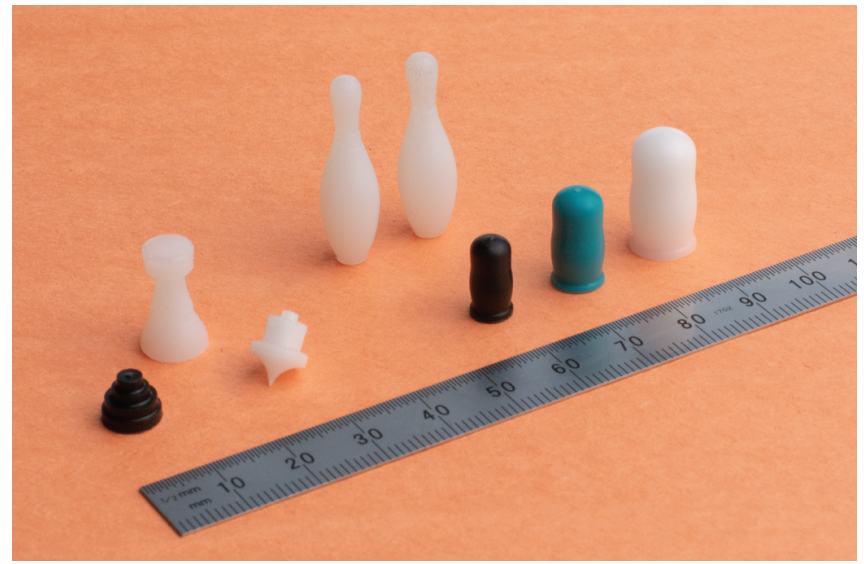

Figure 2. Components made using our system, from right to left: Nesting dolls of three sizes, bowling pins, spinning top, chess rook, and Energy Dome. Ruler scale in millimeters.

tional fabrication and design workflows [45, 41], and extending digital fabrication to new contexts $[11,42]$. Additionally, emergent critical works have directly challenged the delegative interactions of existing digital fabrication workflows [7, 43].

Our primary focus in this work is feedback - fabrication tools are not only output devices for creating physical artifacts, but input devices that can and should inform the user about the state of their work and the capabilities of the system. Specifically, we focus on how computationally defined haptic feedback can be uniquely utilized as a modality for manually-operated tools. Computational interventions need not be limited to glorifying and recreating the sensations lost when transitioning from manual to digital fabrication, but can mediate new user experiences that heighten sensibilities and expand capabilities. This focus on digitally mediated haptic feedback aims to leverage our existing capacity for nuanced and skilled physical actions [18], rather than focusing exclusively on display centric modalities. Broadly, we seek to create more lucid fabrication tools: those which respect our capacity for embodied knowledge to skillfully and keenly respond during the process of fabrication, by allowing design intent to be fluidly expressed and feedback to be clearly understood. Though these ideas around more lucid experiences are generally applicable, we examine these ideas in specific detail through a lathe.

\section{Turn-by-Wire}

Lathes are fabrication tools in which the material being formed is continuously rotating, and tools move about the spinning material to add, cut, or deform it to the desired shape. The act of working with a lathe is called turning, which is referenced by our title. Lathes are historically as well as practically significant and often referred to as "mother tools" [12, 37]. They have endured because of their unique ability to create cylindrical geometries (Figure 2).

Lathes are found in diverse contexts, exhibiting varying levels of handedness in how the tool is used. In the case of a ceramicist's pottery wheel, hands are directly used as tools. One step removed from this directness is a wood lathe, where sharp tools are mounted on handles, giving the crafts-person greater leverage over the cutting forces and process. Going one step further is a manual lathe, where cutting tools are mounted to a two-axis gantry, and the tools are moved through the use of handwheels - large rotary knobs which transmit the rotational motion applied by the user to linear motion of the two axes through a mechanical transmission. In this third style of lathe, the physical transmission mechanism between the user and the gantry acts as rudimentary mediator between the actions of the user and the actions of the lathe. This decoupling between user action and machine action augments the capabilities of the user by improving safety, productivity, and accuracy. It also reduces the directness of how the user works with the material, but the tactile sensations of working with the material are not entirely lost. Forces felt by the cutting tool are attenuated through the mechanical transmission. Fourth and lastly, CNC control takes this decoupling to an extreme, and all actions performed by the lathe must be specified through a series of software packages. This CNC workflow is not specific to lathes, but representative of the workflows for many other digital fabrication tools today.

Situated between the mechanical couplings of a manual lathe, and the disembodied control of a CNC lathe, Turn-by-Wire utilizes "drive-by-wire" handwheels to directly but digitally couple the user and the tool. A user directly moves the cutting tool through the use of the handwheels, but how the cutting tool moves is entirely software defined. The same is true for the forces that are felt through the handwheels; these handwheels act as the locus of interaction not only for expression, but also feedback. This "by-wire" infrastructure facilitates our primary contribution: the concept and implementation of a richer set of haptic feedback that mediates a user's interaction with a fabrication tool. These haptic interactions are not limited to literal representations of feeling a desired model or imitating the forces experienced by a tool. Instead, we leverage haptics to empower users to communicate intent, perceive state, and acquire technique during the fabrication process. For example, consider "snapping-to" a haptic guide, amplifying the forces felt by a delicate cutting tool, or learning a new technique through haptic guidance. We implement these interactions and more through a system consisting of an electro-mechanical lathe, force feedback handwheels for physical input/output, and a graphical user interface for visual input/output (Figure 1). In this paper, we detail the system's design, implementation, and evaluation, as well as how its embodied ideas can be more broadly applied.

\section{RELATED WORK}

This project is situated primarily within the literature of interactive fabrication and augmented hand tools, and is informed by historical and critical perspectives for digital fabrication.

\section{Augmented Hand-Held Tools and Interactive Fabrication}

Researchers have broadly explored augmented hand-held tools in contexts as diverse as sculpting [51,35], spray-painting [39], on-body fabrication [11], wood routing [36], and many more in industry and academia, as surveyed in [52]. In addition to stand-alone smart tools, ecosystems have been proposed [38]. By augmented, these projects often mean that the tool has knowledge about a desired digital model, and has sensing 
and actuation to guide the user towards that goal. However, not all projects anchor the user so explicitly to a predestined outcome: [50] created scissors which responds to conductive ink drawn by the user, Protopiper allowed users to build large sketch models using a hand-held tube extruder [2], and [49] developed a drawing system that can guide a user's pen with a magnet. Interactive systems embodying critical perspectives challenge and invert these goal-oriented workflows more directly; in these alternative envisionments, users manually follow instructions typically sent to a $3 \mathrm{D}$ printer [8, 7], use $3 \mathrm{D}$ printed objects as proxies that scaffold manual fabrication [43], and continuously modify an object during 3D-printing [17]. This project similarly foregrounds open-ended workflows in which computation mediates the process, rather than a particular product, of fabrication.

In addition to considering the workflows that these systems support, the same literature can simultaneously be viewed through the lens of interactions - how does the user guide the tool, and what feedback is communicated about the fabrication task? With hand-held tools, our sense of touch can be uniquely leveraged for feedback. Many projects utilize this quality passively $[51,36,11]$, and the user simply feels forces through the augmented tool as they would a tool without computation. However, [16] began to explore how haptic feedback could allow a user to feel a 3D model, and $[49,50]$ operate similarly by guiding a user's hand with motors and magnets. Though not strictly fabrication, haptics is often suggested as a way to communicate "feel" in tele-operated surgery [34]. Leveraging the "by-wire" control, this work curates a set of haptic interactions that extend beyond the literal and model-centric views of what can be felt through a hand tool.

Fabrication tools need not be hand-held for them to be directly manipulable. For example, Interactive Fabrication explored how embodied input such as physical movement or voice can be interpreted as geometries for fabrication [48, 47]. A digital "drafting table" on the lid of a laser cutter allowed users to quickly create assemblies [25] and folded objects [24], and similar ideas have been explored in the context of woodworking [42] and large scale drawing [21]. These works primarily considered interactions that communicate a desired geometry to a fabrication tool. Exploring feedback rather than control, [27] proposed spatial AR for visualizing real-time cutting forces experienced by a CNC lathe running a pre-defined toolpath. Turn-by-Wire unifies these complementary ideas, simultaneously allowing design intent to be fluidly articulated, while directly conveying information about the state of the work and capabilities of the system.

\section{Haptics}

Diverse haptic feedback modalities are broadly explored in domains such as mobile devices and AR/VR. For example, hand held controllers have been imbued with software defined variable stiffness springs [13], combined force feedback and skin stretch [32], and texture rendering [5]. Additionally, un-grounded force feedback has been implemented through flywheels [4] and asymmetrically accelerated voice coils [6]. This sparse sampling highlights the rich palette of modalities that can potentially be leveraged within digital fabrication.

\section{CNC Machine Tool Interactions}

Since the inception of numeric control in the mid-20th century, machine tool manufacturers have similarly grappled with questions around how to interact with the capabilities of digital fabrication tools. While CAD/CAM pipelines have emerged as the dominant workflow for generating toolpaths which control machines, alternatives which lower the barrier to entry continue to be explored. An electronic handwheel accessory for CNC lathes was recently proposed [14], though it offers little beyond imitating manual machining. Conversational programming - GUI "wizards" for creating toolpaths of commonly used operations - is available from many CNC machine manufacturers $[10,1]$. While possibly easier to learn, this workflow is equivalent to that of CAD/CAM in an important way: an operator first creates a toolpath, then watches idly while that toolpath controls the machine. Many HCI projects in interactive fabrication such as $[42,25,48]$ continue the thread of this broader work. Most interestingly, one of the earliest technologies proposed for controlling NC tools allowed a machinist to define a toolpath by manually cutting the first part. The actions are recorded and replayed for subsequent parts, in a process called Record-Playback [26]. The most important distinction of this workflow is that rather than attempting to tighten the interaction loop of programming and machining, the embodied actions of manually machining is the programming. We build upon this seminal work to further consider how computation can augment and extend a user's capabilities during the process of making.

\section{Interactive Design}

Parallel to the focus on fabrication tools themselves, researchers have explored novel interactions for generating the 3D models that will be fabricated. Here, embodied interactions is similarly suggested as a strategy for navigating the limitations of designing physical objects through GUIs. For example, transferring dimensions between real and virtual environments was enabled through measuring tape [19] and actuatable calipers [44]. Copying real world objects as input for a CAD model was implemented in systems such as CopyCad [9] and MixFab [46]. Augmented with sensing, drawings on paper models [40] and construction kits [20,3] also bridged the physical/digital divide. Haptics has also been suggested for augmenting input for computer aided design [31] and manufacturing [22]. These works take a compartmental view of making: a designer leverages embodied interactions to define a 3D model, which is then delegated to a fabrication tool.

Other works resist this partitioning. Systems have been created to investigate the concurrent fabrication of a low fidelity model [23] in parallel with the user's creation of a CAD model $[29,28,30]$. Systems have also enabled bi-directional fabrication, where a physical model can be continuously edited by a machine [41], user, or both [45] after it is first created by a fabrication tool. We similarly investigate workflows in which the division between design and fabrication is amorphous. The physical act of machining a part using Turn-by-Wire, mediated by interactions for expression and feedback, is also the process for designing a model in-situ of the manufacturing constraints required to fabricate it. 

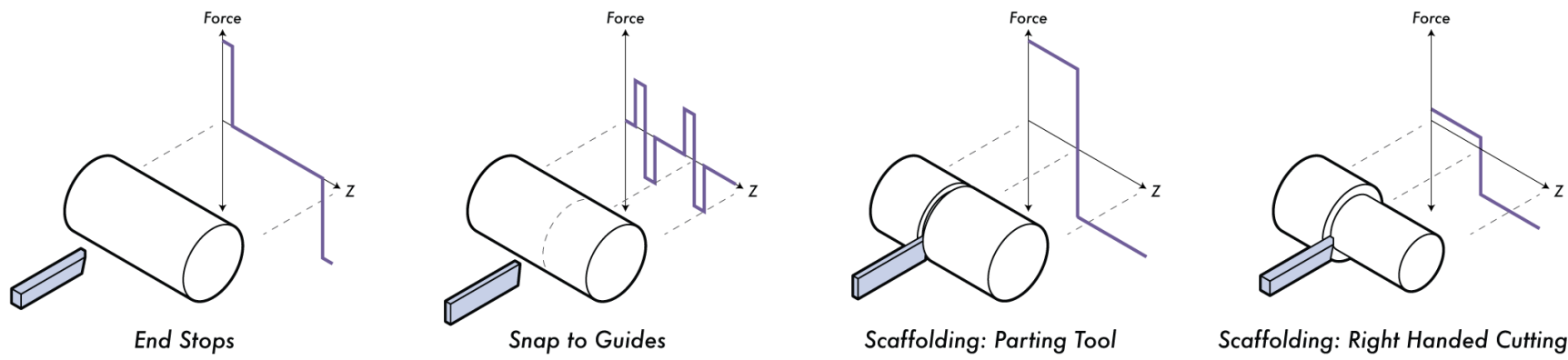

Figure 3. To illustrate the forces a user feel while using our system, we qualitatively plot the force with respect to the z-position of the tool. The cutting tool is shaded in blue, and the cylinders represent the stock material to be cut. From left to right: Feeling the end stops, snapping to guides, haptic scaffolding while using a parting tool (tool cannot move in the z-axis after it has plunged into the material), and haptic scaffolding while cutting with a right handed tool (tool can only move right to left).

\section{INTERACTING WITH TURN-BY-WIRE}

Our system leverages the direct but modulated control of digital fabrication tools for three primary goals: (A) perceiving state, (B) scaffolding, and (C) communicating intent. We first describe a short example of how these features are used in the context of fabricating a component, then discuss these interactions in detail with respect to their primary goals.

\section{Using Turn-By-Wire to cut a simple shape}

Our user needs to make a cylindrical component for the robot she is building. To do so, she selects a piece of stock material of the appropriate length and diameter, and loads it into the lathe. Through the user interface, she inputs the dimensions of the stock material, establishing the initial correspondence between virtual and physical material. To create the outer diameter of the geometry, the user wants to use the righthanded cutting tool, a common tool used for general-purpose cutting. She selects the tool from the GUI, and the position control of the lathe reciprocates from her to the machine as the system moves automatically to complete a tool change; handwheel controls are locked out while the machine is moving autonomously. When the tool change is complete, the user regains control of the lathe through the handwheels.

Using the 2D visualizer in the GUI, she sets up a guide-line to her desired dimension before starting the cut. Outside of the bounds of stock material, she turns the radial axis handwheel until she feels the handwheel click into the guide location that she has set on the GUI. She nudges the handwheel from side to side and feels a gentle centering force. Satisfied that the diameter is precisely set, she turns on the main motor to begin cutting.

She begins her cut by moving the tool along the z-axis of the spindle. As soon as the cut begins, she feels the resistance in the handwheel. Through her experience working with the machine, she knows that this amount of force corresponds to a safe cutting speed. As the physical material is being cut, the virtual material's geometry is simultaneously updated. Toward the end of this operation, she notices that she can no longer advance the z-axis any further - there is a virtual wall blocking her way. She turns off the main motor to examine the setup more closely. She sighs a breath of relief; it has been a while since she has used her lathe, and she had almost moved the $\mathrm{z}$-axis into the frame of the lathe.

A few more operations later, our user finishes the component. Knowing that it would be good to have a backup of this part, she sets up the lathe to automatically duplicate the component, using the toolpath she just generated through directly interacting with the machine.

\section{A - Perceiving State}

The force of a cut is felt through the handwheel by electronically braking the motors. In addition, these forces can be amplified or attenuated depending on the task at hand. For example, a user might might choose to amplify the force on a delicate cutting tool to give themselves a more nuanced feel of how the cut is progressing. Physical attachments for manual machines are often created to accomplish this same task for small diameter drills. Rather than having the first indication of a broken or failing part be the failed part itself, a user of this system immediately feels that something might be awry.

\section{B - Scaffolding}

While haptics may guide the user to better understand the state of the machine, we also consider how it can be leveraged to scaffold novices into a new domain, or for experts to learn new techniques. We create two haptic interactions to conservatively bound the user within safe operating conditions: limit stops and technique scaffolding. Users can toggle these features through the GUI to adapt the tool to their skill level. While these interactions restrain the allowable input of the user, they also seek to foster an environment where open ended exploration is encouraged and de-risked.

\section{Limit stops}

The position of the lathe with respect to the joint limits is known at all times. When the user begins to approach the travel limits, a virtual spring renders a repelling force, indicating to the user that something limiting the motion of the lathe is close by. When this feature is enabled, the chance of the tool crashing due to an error in setup is dramatically reduced.

\section{Technique Scaffolding}

Lathes employ various types of tools for different operations. Similarly varied are the techniques for using those tools: pre- 
ferred direction of cut, where or how to initiate or exit a cut, etc. While some of these techniques are a matter of whether or not the tool will cut at all, others are relevant for producing tighter tolerances or better surface finishes, and others yet are matters of personal style. When technique scaffolding is enabled, we restrict the user's input to the lathe through haptic feedback. One example of a strict rule check for technique is disallowing a user from moving a parting tool side to side after it has plunged into the material (Figure 3 center right). Doing so may damage or break the tool, depending on the material being cut. This restriction is indicated to the user by rendering a strong spring centering the $\mathrm{z}$-axis handwheel after the cut has been initiated, which is released only when the user has backed the tool out of the material.

\section{C - Communicating Intent}

Complementary to features oriented toward feedback, we create three interactions that allow design intent to be expressed more concisely. These interactions incorporate affordances typically associated with digital editing to augment the experience of physical fabrication.

\section{Snap to Guide}

Virtual guides (detents) are added and visualized through the GUI (see Figure 7), and felt in the handwheels. Without visually interfering with the component being made, we afford a subtle mechanism for the user to track their current location in space.

\section{Virtual Tools}

By using a digital rather than mechanical coupling between handwheel and lathe, we can adjust the "transmission ratio" between the handwheel's rotation and the lathe's motion in software. Rather than having the z-axis handwheel move the lathe only along the z-axis, this handwheel can be remapped to cut along a taper or radius, as opposed to coordinating two handwheels manually or by using a context-specific jig. Custom defined profiles can also be used. In the UI, this remapping is conceptualized as "virtual tools". The dimensionality of the task is effectively reduced to highlight the most salient aspects about the design.

\section{DRY: Don't Repeat Yourself}

Copy and paste is an ubiquitous feature of digital tools, allowing users to cache and pattern motifs. We extend this concept to physical fabrication: once a object has been made by the user once, she can replay her actions with minimal effort, as in the scenario above. The design intent only needs to be communicated once, as all user inputs can be recorded.

\section{IMPLEMENTATION}

In this section, we describe the hardware, electronic, and software infrastructure that underlies our system. At a high level, the interactions are implemented using microcontrollercontrolled hardware devices (lathe and handwheels), whose collective behavior is coordinated by a web-application that communicates to each hardware device through UART Serial. This architecture allows us to abstract the implementation of coordinated behaviors into a more high level programming language, while maintaining the timing reliability of microcontrollers for control loop based interactions, such as haptic
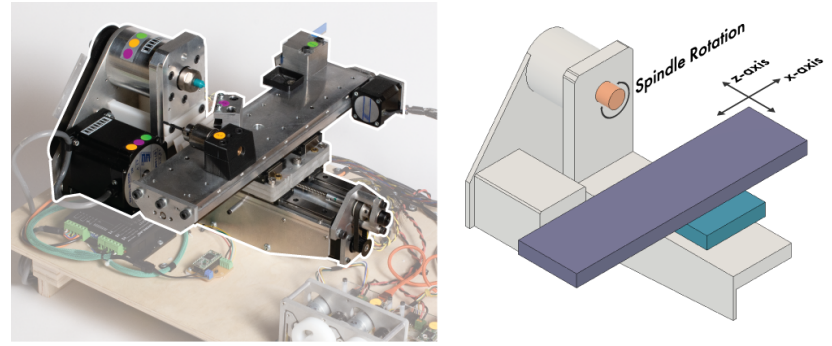

Figure 4. Left: The lathe is highlighted in a white outline to distinguish it from the background. Geometries to be cut are mounted to the spindle using a collet, which is currently holding a blue workpiece. Right: schematic view of the lathe. Rotating spindle in orange, $x$-axis in purple, z-axis in blue.

rendering. We highlight implementation details, discuss design motivations, and give a concrete example of how the system works together to operationalize the snap-to-guide functionality.

\section{Lathe}

We designed and fabricated a custom desktop lathe for our system. The lathe's structure is constructed primarily from 6061 Aluminum, using a combination of CNC machining, hand machining, and abrasive waterjet cutting. For ease of integration and assembly, we use linear profile rails to support the motion of the gantry. Stock material of up to $50 \mathrm{~mm}(\sim 2$ inches) in diameter can be cut. A schematic view of the lathe can be seen in Figure 4.

A long X-axis (225 mm 9 inches) allows us to simultaneously mount multiple cutting tools in a "gang tooling" configuration. Because the machine is CNC controlled, the user can easily index between these various tools, effectively using the $\mathrm{x}$-axis of the gantry as an automatic tool changer. The following tools are currently mounted to our machine: a drill (used to create holes), a standard cutting tool (used to cut profiles on the outer diameter), and a parting tool (used for cutting grooves and removing the component from the stock) (Figure 4). These tools encompass many common operations possible on a lathe.

The spindle, which holds the component being fabricated, is driven by a NEMA 34 stepper motor as opposed to a brushed or brushless DC motor. This simplifies precise velocity and position control of the spindle. Though the maximum rotational speed is limited by the use of a stepper motor, respectable torques (approximately $4 \mathrm{~N}-\mathrm{m}$ at 100RPM) are achieved at lower speeds. The lathe's motors are controlled by a microcontroller running GRBL, an open source motion control firmware. G-code and other low level commands are streamed to this microcontroller over UART Serial.

\section{Force Feedback Handwheels}

We designed the visual layout of our two handwheels to reference the arrangement found on a traditional lathe: one larger handwheel for controlling the z-axis, and a smaller handwheel positioned to the side and offset above for controlling the $\mathrm{x}$-axis (Figure 5). In a manual lathe, the handwheels are mechanically coupled to the motion of the lathe, and the user feels the forces required to move the lathe as these forces travel through the mechanical transmission. In contrast, our 


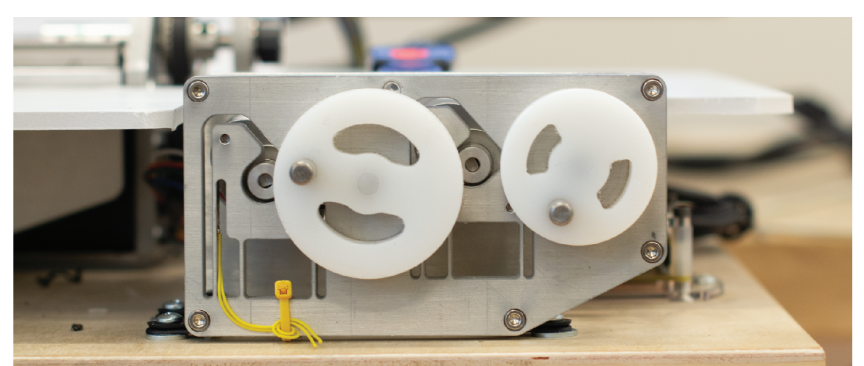

Figure 5. The arrangement of the $X$ and $Z$-axis handwheels references the placement on a traditional lathe.

handwheels are drive-by-wire; they measure position and output force to the user, but there is no coupling between the handwheel and the lathe other than the ones we design and implement in software. This architecture is the basis for all of the interactions we have developed.

\section{Mechanical Design}

Each handwheel subassembly consists of a handwheel mounted to a rotary shaft, a brushed DC motor with encoder, and a belt drive transmission coupling the DC motor to the handwheel shaft. A frame, consisting of two waterjet aluminum plates separated by spacers, house these components (Figure 5). The handwheel motors are mounted to flexural stages monolithically integrated within the frame; the thin flexural elements allows the motor to deflect slightly when the transmission is under load. In future work, we discuss how this deflection can be measured to directly calculate the force experienced by the user.

\section{Electronic and Firmware}

Electronics and firmware is modularized by controlling each handwheel independently with a dedicated microcontroller. A custom printed circuit board interfaces with the encoder, motor, and other I/O. Each handwheel microcontroller exposes a UART Serial interface for commanding low level actions such as querying the current position, electronically braking the motor, or rendering a virtual spring at a desired position. More complex interactions are orchestrated by the web application.

\section{Haptic Control Loop}

The handwheels utilize two types of haptic feedback, electronic braking (passive) and virtual springs (active). For stability and ease of implementation, electronic braking is used to render continuous cutting forces. The cutting force rendered is proportional to the volume of material removed by the tool, which is calculated using the virtual model and tool. Active feedback is used for discrete features of interest such as virtual walls and detents, and is implemented using an impedance (measure position, output force) type haptic controller [15]. The control loop only renders one type of haptic feedback at once, based on a hierarchy of the haptic interactions. For example, feeling a virtual guide takes precedence over feeling the force of the cut; when the user is cutting material but enters the range of a guide, the passive braking is disabled, and the user only feels the centering force of the guide. Though not implemented, an admittance type haptic controller (measure force, output position) could be used by measuring force through the flexural stage.

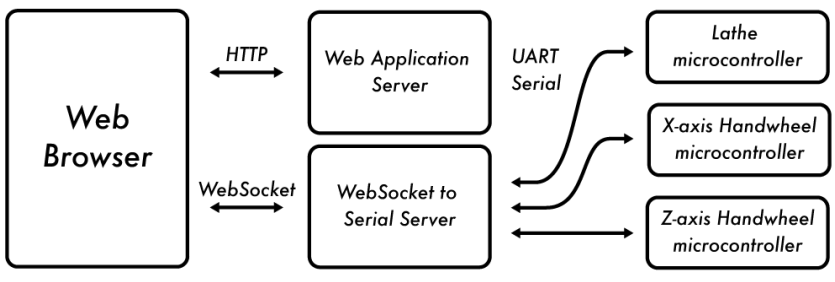

Figure 6. Left: User facing UI is rendered in a web browser. Center: Two web servers are used, one to serve the web application, and the other to act as a WebSocket to UART Serial bridge. Right: Three microcontrollers interface with the hardware.

\section{Orchestrating Hardware through Web Applications}

Using web applications to manage interactions between hardware devices affords two main benefits: the Turn-by-Wire system can be controlled by any device with a USB port and access to a web browser, and code orchestrating interactions between hardware devices is maintained in JavaScript rather than in microcontroller firmware. Turn-by-Wire uses two locally hosted web servers to coordinate interactions between the lathe and handwheels (Figure 6 center). The immediate interface to the hardware serial connections is a Serial to WebSocket bridge implemented using Tornado, a Python web framework. The UI is displayed on a computer monitor located adjacent to the lathe, and is served through a Sinatra DSL web server. We use Paper.js to render the top-down visualization of the lathe (Figure 7). The majority of the application logic is handled by front-end Javascript.

\section{GUI Design}

The GUI is designed to complement the specialized input modality of the handwheels. While the haptic handwheel user interface is well suited for controlling position and outputting force, not all interactions with the fabrication tool should be expressed through the haptic handwheels. In particular, actions such as selecting a tool to cut, or inputting the size of the stock material are better represented through a standard GUI. The elements of the GUI are visually grouped by function; Figure 7 indicates these regions with overlaid colored boxes. At the top of the page (purple outline) is a row of machine settings such as loading new material, or toggling on/off the technique scaffolding. The large pane in the center (blue outline) is a top down $2 \mathrm{D}$ visualizer view of the moving tool and component being cut. The geometry of the part being cut is also updated live, using Boolean intersection operations between the stock material and the cutting tool. To the right of this pane (orange outline) is where users select the cutting tools.

\section{Maximizing responsiveness of the system}

The end-to-end latency through the software pipeline in Figure 6 , as measured by the time between sending a command to a handwheel from the front-end JavaScript and receiving the response, is on the order of $10-20 \mathrm{~ms}$. During operation, we are able to maintain a frame rate of 40-60FPS. The limiting factor is the recurring draw event for Paper.js, which slows down as more cuts are taken from the rendered polygon. Though this refresh rate is variable, the microcontroller firmware - 

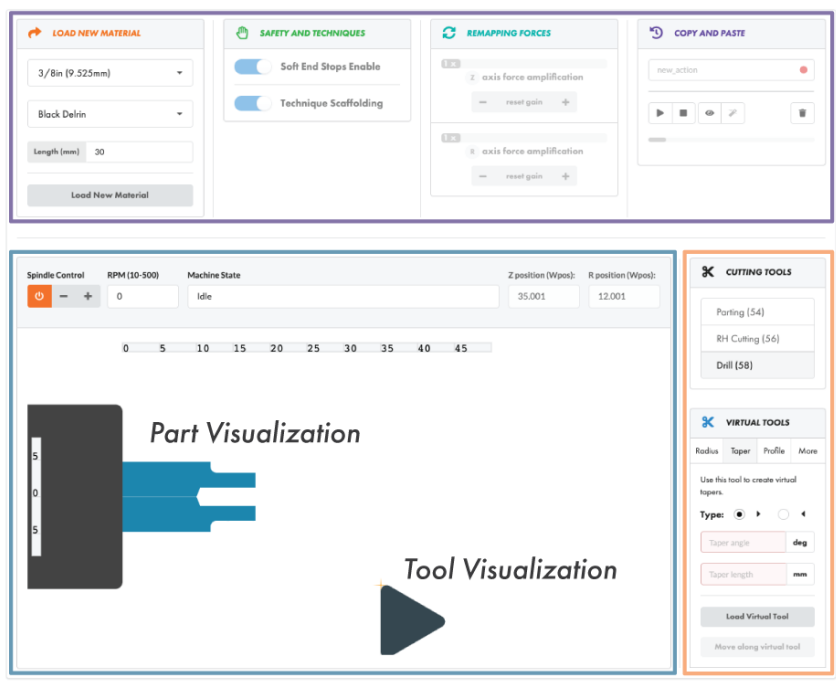

Figure 7. Not all actions can be specified through the haptic handwheels. We use the flexibility of a GUI to complement the handwheel input. Colored lines outline various groups of the UI. Purple (top): tool settings such as toggling on or off haptic scaffolding. Orange (right): interface for choosing cutting tools, both real and virtual. Blue (left): top down 2D visualizer of the lathe, stock material, and cutting tool. Stock material visualization updates as cuts are taken.

which controls the haptic control loops — is always running "real-time" under timer interrupts.

Even when the software latency is reduced, the overall responsiveness of the handwheel to lathe coupling also depends on the latency of the hardware itself (e.g. the delay in accelerating the lathe's gantry). We additionally adjust our system through the following strategies. First, we tune the acceleration of the stepper motors near the maximum allowable limit, which is bounded by the motors' available torque. Combined with lowering the maximum allowable velocity, these changes allow our lathe to rapidly come up to speed - from stationary to maximum velocity in under 100 milliseconds. Next, because the "transmission ratio" from handwheels to lathe is defined in software, we qualitatively find a satisfying balance point between fine grained movements and maximum lathe velocity. Qualitatively, these strategies result in highly responsive interactions.

\section{System Walk-Through: Adding a Guide}

We detail how our system coordinates the different layers of software and hardware to enable the snap-to-guide interaction. Users add guides through drag and drop interactions starting from the rulers located at the top and left of the 2D visualizer canvas. The guide is visualized as a reference line through the GUI and saved on the front-end (Figure 7). Simultaneously, the web application issues a command to the corresponding handwheel's microcontroller to add a virtual detent at that location. This message travels through the WebSocket and is routed to the appropriate hardware serial device by the WebSocket-to-Serial server. Information about the guide location is redundantly stored on both the firmware of the handwheels, as well as in the JavaScript. The handwheel microcontroller uses this position when executing the real-time haptic control loop. Because of the redundant data storage, the microcontroller is not dependent on data from the web application during this low-level control loop.

When the user rotates the handwheels, the corresponding microcontroller streams location update messages to the web application. The web application applies the virtual transmission to these position messages to calculate the desired lathe position. Subsequently, the desired position is sent to the lathe via a G-code command, and is used to update the GUI.

The user feels a centering force through the handwheel when their tool approaches a guide. To achieve this, the guide location is used as the desired position for a simple proportionalderivative (PD) controller by the handwheel microcontroller. As a result, the motor attached to the handwheel is actuated to maintain the handwheel at the desired position. The gains of the PD controller are chosen such that the user will feel the handwheel being pulled toward the guide location, but can insist to move the handwheel away from the desired position. When the user moves the handwheel far enough from the guide, the haptic detent is deactivated. The "feel" of the detent can be modified by tuning the PD controller, which can be interpreted as the properties of the virtual spring.

\section{USER STUDY}

We focused our evaluation toward understanding how the modulated haptic feedback might support users during the process of fabrication. Specifically, the user study was designed to probe how each of the individual haptic features, as well as the collective workflow supported by the system, would be used and received.

\section{Participants}

We recruited five expert participants ( 3 male, 2 female) from the staff of an educational makerspace. This targeted sampling was motivated by the unique lens that experienced fabricators can lend. For example, though some of the interactions we evaluate (e.g. technique scaffolding) are especially relevant for novices, expert participants are uniquely positioned to articulate why these interactions are or are not useful by contrasting them with their deep experience with manual and CNC tools. As both experienced practitioners and instructors, these participants can also reflect on the types of difficulties faced by novices when they first approach different types of fabrication tools. Lastly, we hypothesized that these experienced users would span a broad spectrum of informed preferences towards fabrication workflows and tools, and could potentially bring these varied opinions to bear when discussing the tool from the perspective of workflows.

Information about users' fabrication background was collected through the recruitment questionnaire. P1 is a fabricator who specializes in industrial and furniture design. P2 is a glass sculpture artist, and P3 is a trained architect and fabricator. P4 is a wood sculpture artist, and P5 is a roboticist who designs and fabricates combat robots. All participants are skilled designers and fabricators with at least ten years of experience in their respective domains. Though all participants incorporate $\mathrm{CNC}$ and hand tools in their practice, $\mathrm{P} 1$ and $\mathrm{P} 2$ lean more towards hand tool usage, while $\mathrm{P} 4$ is more $\mathrm{CNC}$ tool oriented. 

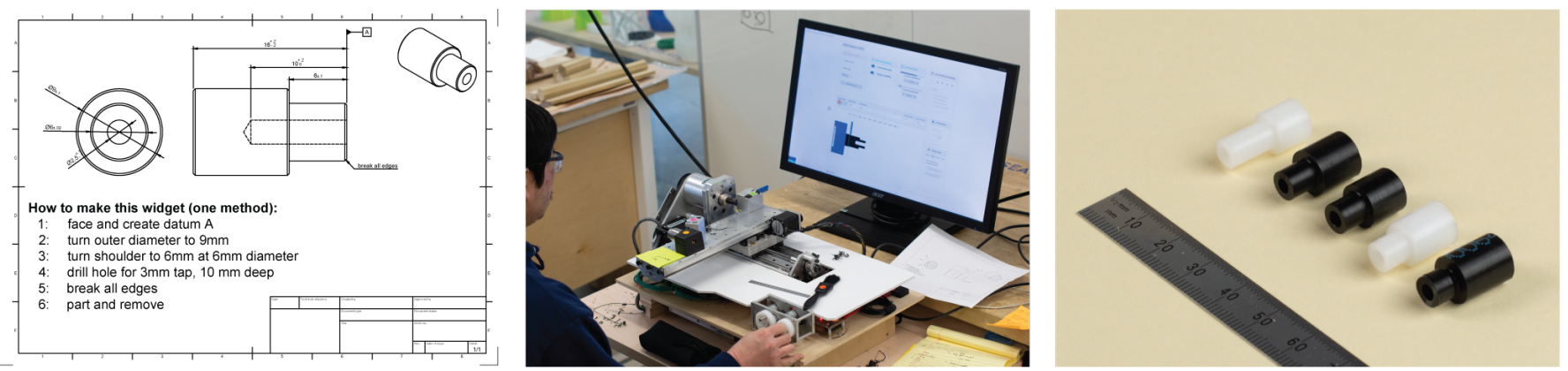

Figure 8. Left: Dimensional drawing given to users during the study. Many of the primary operations possible on a lathe are required to fabricate this component. By design, users also encounter all haptic features while fabricating this component. Center: User study setup with lathe, handwheels, and monitor to display the GUI. Right: Widgets made by the five users during the user study. We suggested to the participants to focus primarily on familiarizing themselves with the tool and haptics, rather than achieving perfect dimensions for the outcome.

P3 and P5 are centrist in terms of preference towards manual or CNC tools. Some of the participants are also responsible for teaching and developing curriculum for $\mathrm{CNC}$ machines, such as wood routers and metal milling machines.

\section{Procedure}

Users participated in a one-hour study and were compensated with a US $\$ 20$ gift card. The study began with reviewing and signing research consent documents (10 minutes), followed by an introduction to the various elements of the system and the haptic interactions that are supported (15 minutes). The primary portion of the study was a fabrication task (20 minutes) designed to expose participants to all of the haptic interactions as they turned a component using the system (Figure 8 center). After users completed the component, we conducted a semistructured interview in the remaining time. Following the in person portion of the study, users anonymously completed an online questionnaire.

We tasked all participants with fabricating a specific component. Though we are interested in having future work engage participants in more open ended tasks, we chose this more controlled evaluation first to focus more narrowly on the efficacy of the haptic feedback features. After the system introduction, a printed copy of a dimensional drawing (Figure 8 left) and a finished example of the component where shown to the users. The supplied drawing included desired dimensions as well as a suggested ordering of cuts that can be taken to create the geometry. We prompted users to primarily focus on familiarizing themselves with the tool and experiencing the haptic feedback, rather than getting each of the dimensions perfect. Users created the component using acetal, an engineering thermoplastic with good machinability.

The design of this component was inspired by our on campus Mechanical Engineering student shop, where members make a similar component in the final hands-on portion of machine shop training. Many of the primary operations possible on lathes are required, including (1) turning (cutting) the outer diameter, (2) facing (cutting the end of the stock material), (3) drilling, and (4) parting (removing the component from the stock using a thin cutting blade). All three tools currently mounted to our machine are used, across six operations. By design, users encountered all haptic interactions while fabri- cating this component, for example, setting up virtual guides to drill at the correct depth.

\section{USER STUDY RESULTS}

All participants completed the component within 15 - 20 minutes (Figure 8 right). In the post-task interview, users expressed overall excitement around this type of tool. This result was corroborated by the anonymous exit survey - participants responded positively to a set of five-point Likert scale questions relating to both the specific interactions as well as the overall experience (Figure 9).

\section{Approaches to making the widget}

Despite the common end goal, the task allowed for some amount of freedom in how users explored the system. Only $\mathrm{P} 2$, the user with the least previous familiarity with lathe tools, followed the ordering of operations suggested by the drawing. Limit stop and technique scaffolding were enabled by default at the beginning of the study, and two participants (P1, P3) disabled this feature when they wanted to make the final "parting" cut closer to the origin, as the software endstops were set conservatively. The feature was engaged during the rest of the study. Feeling the force of cut was also enabled by default for all participants and could not be disabled. Encouraged by the dimensioned drawing, all participants used the snap-toguide feature extensively to mark locations of interest, such as the outer diameter, and depth of the hole.

\section{User perceptions of haptic interactions}

In the anonymous exit survey, users indicated strong positive reactions towards the haptic interactions we implemented (Figure 9 right). All participants responded with "Agree" or "Strongly Agree" in response to all haptic feedback features. The haptic snap-to-guide and technique scaffolding were the most and least positively rated features respectively.

\section{Snap-to-guide}

Snap-to-guide was an especially compelling feature for many of our users ("Strongly Agree": 4, "Agree": 1). In a very lightweight way, this feature captured one of the main characteristics users look for in CNC tools - automatic accuracy.

P5 [The most memorable part of the tool was] the ability to set the limits so you don't cut too far and actually helps you make the exact right dimensions that you want. That sets it apart from any 

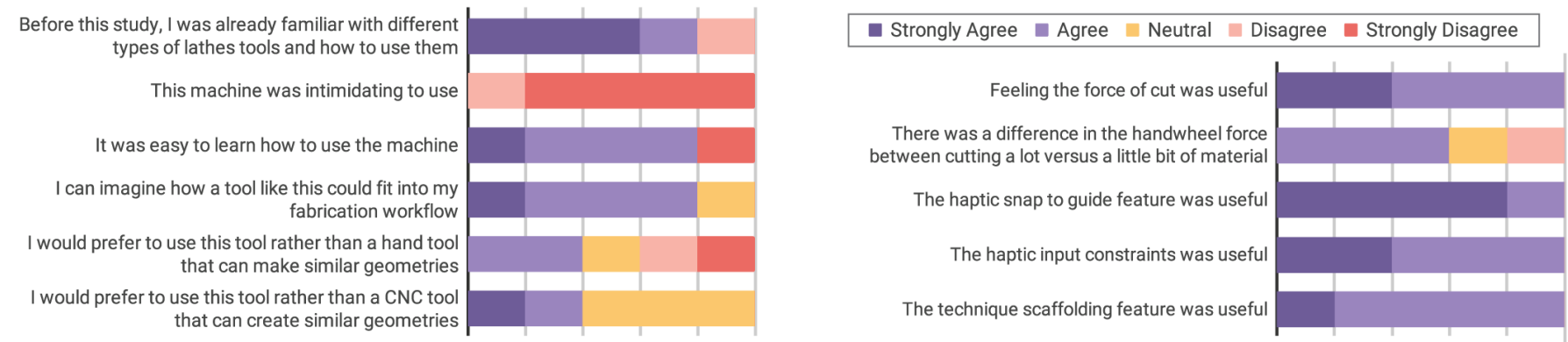

Figure 9. Anonymous questionnaire responses. Left: responses to high level questions around the overall experience. Right: responses to specific haptic interactions mediated by our system.

other machine. Just manual machine or DRO (digital read out) doesn't physically stop you from going past those limits.

While maintaining the directness of physical fabrication, our tool overlays capabilities typically associated with digital editing. Users also commented on the the satisfying "feel" of snapping to the guide lines.

P4 I want to have an excuse to come back on this tool again because I loved those snap lines and that feeling you get, ... like when you're actually designing in CAD and you snap to align it to the right line and it feels good. Yeah, well this is that physical sensation.

\section{Scaffolding through haptics}

Haptic scaffolding received the least positive feedback in our exit-survey, however all participants were still overall positive: "Strongly Agree": 1, "Agree": 4. We hypothesize that this is related to our users' high baseline familiarity with lathes. P2, the most hand tool oriented participant, uniquely conceptualized our system as a CNC machine to teach material sensibilities.

P2 It's CNC to teach you material consideration... how to feel material.

\section{Feeling the force of cut}

When asked about whether they could feel the difference between cutting "a lot" versus "a little" bit of material, responses ranged from "Agree":3, "Neutral": 1, to "Disagree": 1 . This spread may have resulted from the fact that the force of cut was tuned for the easily machinable plastic used during the user study, in addition to the ambiguity of what "a lot" or "a little" material meant for the user. In the anonymous exit survey, one participant wrote, "When doing actual cutting, especially with the plastic, it felt like butter." In other words, the material chosen may have been too easy to cut to discern any important differences in cutting force.

Despite this, all participants perceived being able to feel the force of cut as a useful feature ("Strongly Agree": 2, "Agree": 3 ). During the interview, P4 directly commented on the ability to "feel" the machine.

P4 My favorite thing about this so far is that machine feel, [it] is so difficult to communicate to people like what it should feel like.

Volumes of tacit knowledge [33] are embodied in hand tool usage. While phrases such as tightening something "monkey tight" versus "guerilla tight" try to capture the subtleties of touch, there remains ambiguity that must be calibrated through experimentation and experience. Turn-by-Wire short circuit this process by creating an environment in which the feel of a fabrication tool can be communicated directly.

\section{Limitations}

While being able to remap forces is what enables many of our system's interactions, the software mediated nature of the force feedback is also a source of ambiguity. P4 contrasted using our tool with the experience of using a hand plane for woodworking.

P4 If I'm trying to do a hand plane and it's not cutting, it's either the way I've set it up or my technique cause I'm cutting up the wrong way on the grain. But here there is that kind of black box with a question mark on it in between me and the operation of the tool.

Another limitation of our implementation is the spatial separation between the visualization of the part being cut, and the physical part itself. During the interview, users commented on wishing that they had more control over the placement of the screen (P5), and having to switch between looking at the screen versus the machine $(\mathrm{P} 2)$.

\section{User perceptions of workflow}

Responses to the workflow were overall positive (Figure 9 left). In the anonymous exit survey, no user found the machine "intimidating to use", and most found it "easy to learn". While this is likely related to our users' breadth of fabrication experience, two of our users reported during the interviews that our system drastically contradicted their initial expectations of what learning the tool would be like.

P1 It was a lot more comfortable than I expected because in the beginning you told me it's kind of like a CNC machine that you guys are building and I thought ... the learning curve is going to be bigger than just what happened today.

P2 I think the most memorable part was just sort of the ease of use... I mean, honestly I had some hesitation in because my familiarity is very minimal with a metal lathe... I don't think that is easy to achieve with CNC in terms of making people feel confident almost immediately.

P4 I love the idea that it's taking the scariest thing in a typical prototyping shop and making people feel a lot better about using it. 
The haptic feedback in the handwheels give users an additional, and literal, handle to better understand and grasp the system.

\section{Is Turn-by-Wire a hand tool or CNC tool?}

This question prompted diverse responses in our interviews. $\mathrm{P} 1$ and $\mathrm{P} 3$, reflecting on the direct and handed interactions required to operate it, felt that our system was closer to a hand tool. P5 leaned towards CNC control, noting that under-thehood, the system is entirely computer controlled and can be programmed to run autonomously without modification. P2, the most hand-tool centric participant, felt that Turn-by-Wire is definitely a CNC tool, but "very closely maybe bridges those two... it's as easy to use as a hand tool while embodying the components of a CNC". P4 strongly felt that it was "right down the middle", elaborating that one aspect which prevented the system from feeling entirely like a hand tool is the ambiguity that the by-wire control introduced. Users' varied interpretations of their experiences echo our own intentions for tools like Turn-by-Wire, that they occupy a liminal space between hand tools and $\mathrm{CNC}$ tools in which users can engage with the capabilities of a CNC machine fluidly and directly.

\section{DISCUSSION AND FUTURE WORK}

\section{Combining Visual AR with Haptic AR}

The haptic experiences we develop can be thought of as a kind of augmented reality - the sensations a user feels while cutting are curated and augmented, instead of a literal portrayal of the induced forces. We envision blending these haptic experience with visual augmented reality. This combination can be used to build rich experiences for scaffolding, for example to render both the look and feel of cutting difficult geometries or materials. For maximum safety, entirely virtual materials can be "loaded into" the lathe for experimentation and practice (Figure 10). Softer test materials can also be made to feel like those that are more difficult to machine, to reduce the amount of graphic rendering required to create an immersive experience. From a more practical standpoint, an AR visual display would also address the physical separation in the current system between the lathe and digital UI. Hands-free AR interfaces can be implemented through traditional head-mounted devices or fixed tablet computers. Tablets in particular can be adapted to the form factor of a chip guard, which is typically a piece of clear plastic that shields users from cutting debris.

\section{Experiencing How Cuts Shouldn't Feel}

Many of the haptic interactions we introduce in this paper focused on how this modality can be leveraged to ensure that the user is operating the machine safely. Similarly, haptics can also be utilized to show users how certain operations should not feel. Whether through a jittery handwheel, rendering rough textures, or kicking back, the haptics could potentially be leveraged to reflect the tool's own uncertainty about what the user is about to do, or used during training to familiarize users with the limitation of the machine.

\section{Force Feedback from Lathe Motors}

Both handwheel and lathe motor mounts share a similar design, and can be retrofitted with strain gauges (or other displacement sensors) to measure the output torque of the attached motor.

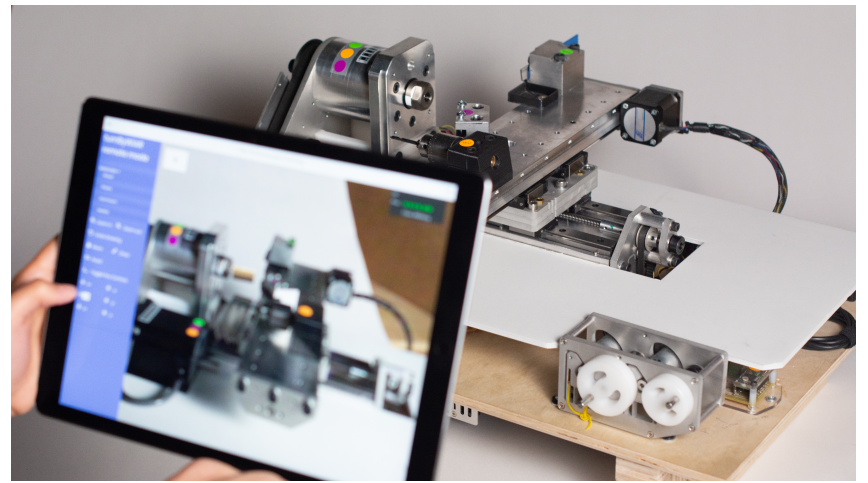

Figure 10. In our AR envisionment, a tablet computer renders a virtual wood material into the lathe (round, tan colored cylinder on screen). No material is physically loaded into the lathe.

This capability can be used to more accurately convey cutting forces to the user through control loops that measure and output specific forces. This is especially important when working with anisotropic materials such as wood, which respond very differently to cutting tools depending on the orientation of the grain. Time variant parameters such as tool wear could also be captured and conveyed.

\section{Other Lucid Tools}

Although this paper focused on lathes, we are primarily interested in how the embedded ideas might change the way we interact with fabrication tools, and how these ideas might generalize and extend to other domains. Many machine shop tools - milling machines, drill presses, grinding machines are also controlled through manual handwheels, readily lending themselves to augmentation through Turn-by-Wire control. Extending similar ideas towards general purpose fabrication tasks will likely require alternative approaches. One possibility would be to utilize exoskeletons, robotics, or other haptic modalities to augment users directly, rather than having the by-wire control be tethered to a particular machine.

\section{CONCLUSION}

Turn-by-Wire curated a set of haptic interactions for expression and feedback that mediates the process of hands-on fabrication. Five expert participants, whose experiences span diverse workflows and domains, used our system and responded positively to the interactions we developed. A lathe was chosen as a vehicle through which to develop these ideas around mediated haptic control. However, we believe that the underlying ideas that guided this work are broadly applicable to fabrication in general, and highlight many avenues for future work.

\section{ACKNOWLEDGMENTS}

We would like to thank the members of the Hybrid Ecologies Lab - Sarah Sterman, Cesar Torres, and Molly Nicholas for their thoughtful and insightful feedback and discussions. We also thank the Siemens Corporation for their generous support of this research. Lastly, we thank the anonymous reviewers for their constructive critique. 


\section{REFERENCES}

[1] Acu-Rite. 2019. MILLPWR G2 Control Systems. (2019).

https://acu-rite.com/millpwr-control-systems.html.

[2] Harshit Agrawal, Udayan Umapathi, Robert Kovacs, Johannes Frohnhofen, Hsiang-Ting Chen, Stefanie Mueller, and Patrick Baudisch. 2015. Protopiper: Physically Sketching Room-Sized Objects at Actual Scale. In Proceedings of the 28th Annual ACM Symposium on User Interface Software \& Technology (UIST '15). ACM, New York, NY, USA, 427-436. DOI : http://dx.doi.org/10.1145/2807442.2807505

[3] David Anderson, James L. Frankel, Joe Marks, Aseem Agarwala, Paul Beardsley, Jessica Hodgins, Darren Leigh, Kathy Ryall, Eddie Sullivan, and Jonathan S. Yedidia. 2000. Tangible Interaction + Graphical Interpretation: A New Approach to 3D Modeling. In Proceedings of the 27th Annual Conference on Computer Graphics and Interactive Techniques (SIGGRAPH '00). ACM Press/Addison-Wesley Publishing Co., New York, NY, USA, 393-402. DOI : http://dx.doi.org/10.1145/344779.344960

[4] Akash Badshah, Sidhant Gupta, Daniel Morris, Shwetak Patel, and Desney Tan. 2012. GyroTab: A Handheld Device That Provides Reactive Torque Feedback. In Proceedings of the SIGCHI Conference on Human Factors in Computing Systems (CHI '12). ACM, New York, NY, USA, 3153-3156. DOI :

http://dx.doi.org/10.1145/2207676.2208731

[5] Hrvoje Benko, Christian Holz, Mike Sinclair, and Eyal Ofek. 2016. NormalTouch and TextureTouch: High-fidelity 3D Haptic Shape Rendering on Handheld Virtual Reality Controllers. In Proceedings of the 29th Annual Symposium on User Interface Software and Technology (UIST '16). ACM, New York, NY, USA, 717-728. DOI:

http://dx.doi.org/10.1145/2984511.2984526

[6] Inrak Choi, Heather Culbertson, Mark R. Miller, Alex Olwal, and Sean Follmer. 2017. Grabity: A Wearable Haptic Interface for Simulating Weight and Grasping in Virtual Reality. In Proceedings of the 30th Annual ACM Symposium on User Interface Software and Technology (UIST '17). ACM, New York, NY, USA, 119-130. DOI : http://dx.doi.org/10.1145/3126594.3126599

[7] Laura Devendorf, Abigail De Kosnik, Kate Mattingly, and Kimiko Ryokai. 2016. Probing the Potential of Post-Anthropocentric 3D Printing. In Proceedings of the 2016 ACM Conference on Designing Interactive Systems (DIS '16). ACM, New York, NY, USA, 170-181. DOI : http://dx.doi .org/10.1145/2901790.2901879

[8] Laura Devendorf and Kimiko Ryokai. 2015. Being the Machine: Reconfiguring Agency and Control in Hybrid Fabrication. In Proceedings of the 33rd Annual ACM Conference on Human Factors in Computing Systems (CHI '15). ACM, New York, NY, USA, 2477-2486. DOI : http://dx.doi.org/10.1145/2702123.2702547
[9] Sean Follmer, David Carr, Emily Lovell, and Hiroshi Ishii. 2010. CopyCAD: Remixing Physical Objects with Copy and Paste from the Real World. In Adjunct Proceedings of the 23Nd Annual ACM Symposium on User Interface Software and Technology (UIST'10). ACM, New York, NY, USA, 381-382. DOI : http://dx.doi .org/10.1145/1866218.1866230

[10] Chris Fox. 2016. A Little More Conversation(al). (Nov. 2016). https:

//www.tormach.com/blog/a-little-more-conversational/

[11] Madeline Gannon, Tovi Grossman, and George Fitzmaurice. 2016. ExoSkin: On-Body Fabrication. In Proceedings of the 2016 CHI Conference on Human Factors in Computing Systems (CHI '16). ACM, New York, NY, USA, 5996-6007. DOI :

http://dx.doi.org/10.1145/2858036.2858576

[12] David J. Gingery. 2014. The Metal Lathe. David J. Gingery Publishing, LLC, Kimberling City, MO, USA.

[13] Sidhant Gupta, Tim Campbell, Jeffrey R. Hightower, and Shwetak N. Patel. 2010. SqueezeBlock: Using Virtual Springs in Mobile Devices for Eyes-free Interaction. In Proceedings of the 23Nd Annual ACM Symposium on User Interface Software and Technology (UIST '10). ACM, New York, NY, USA, 101-104. DOI: http://dx.doi.org/10.1145/1866029.1866046

[14] Haas. 2017. Haas Electronic Handwheels. (2017). https://www.haascnc. com/productivity/product-options/ e-handwheels.html.

[15] Blake Hannaford and Allison M. Okamura. 2008. Haptics. In Springer Handbook of Robotics, Bruno Siciliano and Oussama Khatib (Eds.). Springer Berlin Heidelberg, Berlin, Heidelberg, 719-739. DOI : http://dx . doi .org/10.1007/978-3-540-30301-5_31

[16] Studio Homunculus. 2011. Haptic Intelligentsia. (2011). http://studio-homunculus.com/portfolio/ haptic-intelligentsia-human-prototyping-machine/

[17] Jeeeun Kim, Clement Zheng, Haruki Takahashi, Mark D Gross, Daniel Ashbrook, and Tom Yeh. 2018. Compositional 3D Printing: Expanding \& Supporting Workflows Towards Continuous Fabrication. In Proceedings of the 2Nd ACM Symposium on Computational Fabrication (SCF'18). ACM, New York, NY, USA, 5:1-5:10. DOI : http://dx.doi.org/10.1145/3213512.3213518

[18] Scott R. Klemmer, Björn Hartmann, and Leila Takayama. 2006. How Bodies Matter: Five Themes for Interaction Design. In Proceedings of the 6th Conference on Designing Interactive Systems (DIS '06). ACM, New York, NY, USA, 140-149. DOI : http://dx.doi .org/10.1145/1142405.1142429

[19] Jay Lee, Victor Su, Sandia Ren, and Hiroshi Ishii. 2000. HandSCAPE: A Vectorizing Tape Measure for On-site Measuring Applications. In Proceedings of the SIGCHI Conference on Human Factors in Computing Systems (CHI 'O0). ACM, New York, NY, USA, 137-144. DOI : http://dx.doi.org/10.1145/332040.332417 
[20] Danny Leen, Raf Ramakers, and Kris Luyten. 2017. StrutModeling: A Low-Fidelity Construction Kit to Iteratively Model, Test, and Adapt 3D Objects. In Proceedings of the 30th Annual ACM Symposium on User Interface Software and Technology (UIST'17). ACM, New York, NY, USA, 471-479. DOI : http://dx.doi.org/10.1145/3126594.3126643

[21] Jingyi Li, Jennifer Jacobs, Michelle Chang, and Björn Hartmann. 2017. Direct and Immediate Drawing with CNC Machines. In Proceedings of the 1st Annual ACM Symposium on Computational Fabrication (SCF '17). ACM, New York, NY, USA, 11:1-11:2. DOI : http://dx.doi .org/10.1145/3083157.3096344

[22] Koichi Morishige and Miharu Nakada. 2016. Development of Turning Machine Operation Interface that Uses Haptic Device (Application to Complicated Cutting by Special Byte). Procedia CIRP 57 (Jan. 2016), 649-652. DOI :

http://dx.doi.org/10.1016/j.procir.2016.11.112

[23] Stefanie Mueller, Sangha Im, Serafima Gurevich, Alexander Teibrich, Lisa Pfisterer, François Guimbretière, and Patrick Baudisch. 2014. WirePrint: 3D Printed Previews for Fast Prototyping. In Proceedings of the 27th Annual ACM Symposium on User Interface Software and Technology (UIST '14). ACM, New York, NY, USA, 273-280. DOI : http://dx.doi.org/10.1145/2642918.2647359

[24] Stefanie Mueller, Bastian Kruck, and Patrick Baudisch. 2013. LaserOrigami: Laser-cutting 3D Objects. In Proceedings of the SIGCHI Conference on Human Factors in Computing Systems (CHI'13). ACM, New York, NY, USA, 2585-2592. DOI : http://dx.doi.org/10.1145/2470654.2481358

[25] Stefanie Mueller, Pedro Lopes, and Patrick Baudisch. 2012. Interactive Construction: Interactive Fabrication of Functional Mechanical Devices. In Proceedings of the 25th Annual ACM Symposium on User Interface Software and Technology (UIST'12). ACM, New York, NY, USA, 599-606. D0I :

http://dx.doi.org/10.1145/2380116.2380191

[26] David F. Noble. 1986. Forces of Production: A Social History of Industrial Automation. Oxford University Press, Oxford, England, UK.

[27] Alex Olwal, Jonny Gustafsson, and Christoffer Lindfors. 2008. Spatial augmented reality on industrial CNC-machines. In The Engineering Reality of Virtual Reality 2008, Vol. 6804. International Society for Optics and Photonics, San Jose, California, USA, 680409. DOI : http://dx.doi.org/10.1117/12.760960

[28] Huaishu Peng, Jimmy Briggs, Cheng-Yao Wang, Kevin Guo, Joseph Kider, Stefanie Mueller, Patrick Baudisch, and François Guimbretière. 2018. RoMA: Interactive Fabrication with Augmented Reality and a Robotic 3D Printer. In Proceedings of the 2018 CHI Conference on Human Factors in Computing Systems (CHI'18). ACM,
New York, NY, USA, 579:1-579:12. DOI :

http://dx.doi.org/10.1145/3173574.3174153

[29] Huaishu Peng, Rundong Wu, Steve Marschner, and François Guimbretière. 2016. On-The-Fly Print: Incremental Printing While Modelling. In Proceedings of the 2016 CHI Conference on Human Factors in Computing Systems (CHI '16). ACM, New York, NY, USA, 887-896. DOI :

http://dx.doi.org/10.1145/2858036.2858106

[30] Huaishu Peng, Amit Zoran, and François V. Guimbretière. 2015. D-Coil: A Hands-on Approach to Digital 3D Models Design. In Proceedings of the 33rd Annual ACM Conference on Human Factors in Computing Systems (CHI'15). ACM, New York, NY, USA, 1807-1815. DOI :

http://dx.doi.org/10.1145/2702123.2702381

[31] W. J. Plesniak, R. S. Pappu, and S. A. Benton. 2003. Haptic holography: a primitive computational plastic. Proc. IEEE 91, 9 (Sept. 2003), 1443-1456. DOI : http://dx.doi.org/10.1109/JPROC.2003.817129

[32] C. J. Ploch, J. H. Bae, W. Ju, and M. Cutkosky. 2016. Haptic skin stretch on a steering wheel for displaying preview information in autonomous cars. In 2016 IEEE/RSJ International Conference on Intelligent Robots and Systems (IROS). IEEE, Daejeon, Korea, 60-65. DOI :

http://dx.doi.org/10.1109/IROS. 2016.7759035

[33] Michael Polanyi. 1967. The tacit dimension. Anchor Books, Garden City, N.Y. OCLC: 718091.

[34] Z. F. Quek, S. B. Schorr, I. Nisky, W. R. Provancher, and A. M. Okamura. 2015. Sensory Substitution and Augmentation Using 3-Degree-of-Freedom Skin Deformation Feedback. IEEE Transactions on Haptics 8, 2 (April 2015), 209-221. DOI :

http://dx.doi.org/10.1109/TOH. 2015.2398448

[35] Alec Rivers, Andrew Adams, and Frédo Durand. 2012a. Sculpting by Numbers. ACM Trans. Graph. 31, 6 (Nov. 2012), 157:1-157:7. DOI :

http://dx.doi.org/10.1145/2366145.2366176

[36] Alec Rivers, Ilan E. Moyer, and Frédo Durand. 2012b. Position-correcting Tools for 2D Digital Fabrication. ACM Trans. Graph. 31, 4 (July 2012), 88:1-88:7. DOI : http://dx.doi.org/10.1145/2185520.2185584

[37] L. T. C. Rolt. 1965. A short history of machine tools. M.I.T. Press, Cambridge, MA, USA.

[38] Eldon Schoop, Michelle Nguyen, Daniel Lim, Valkyrie Savage, Sean Follmer, and Björn Hartmann. 2016. Drill Sergeant: Supporting Physical Construction Projects Through an Ecosystem of Augmented Tools. In Proceedings of the 2016 CHI Conference Extended Abstracts on Human Factors in Computing Systems (CHI EA '16). ACM, New York, NY, USA, 1607-1614. DOI : http://dx.doi.org/10.1145/2851581.2892429 
[39] Roy Shilkrot, Pattie Maes, Joseph A. Paradiso, and Amit Zoran. 2015. Augmented Airbrush for Computer Aided Painting (CAP). ACM Trans. Graph. 34, 2 (March 2015), 19:1-19:11. DOI : http://dx. doi .org/10.1145/2699649

[40] Hyunyoung Song, François Guimbretière, Chang Hu, and Hod Lipson. 2006. ModelCraft: Capturing Freehand Annotations and Edits on Physical 3D Models. In Proceedings of the 19th Annual ACM Symposium on User Interface Software and Technology (UIST '06). ACM, New York, NY, USA, 13-22. DOI : http://dx.doi.org/10.1145/1166253.1166258

[41] Alexander Teibrich, Stefanie Mueller, François Guimbretière, Robert Kovacs, Stefan Neubert, and Patrick Baudisch. 2015. Patching Physical Objects. In Proceedings of the 28th Annual ACM Symposium on User Interface Software \& Technology (UIST '15). ACM, New York, NY, USA, 83-91. DOI : http://dx.doi.org/10.1145/2807442.2807467

[42] Rundong Tian, Sarah Sterman, Ethan Chiou, Jeremy Warner, and Eric Paulos. 2018. MatchSticks: Woodworking Through Improvisational Digital Fabrication. In Proceedings of the 2018 CHI Conference on Human Factors in Computing Systems (CHI'18). ACM, New York, NY, USA, 149:1-149:12. DOI : http://dx.doi.org/10.1145/3173574.3173723

[43] Cesar Torres, Wilmot Li, and Eric Paulos. 2016. ProxyPrint: Supporting Crafting Practice Through Physical Computational Proxies. In Proceedings of the 2016 ACM Conference on Designing Interactive Systems (DIS '16). ACM, New York, NY, USA, 158-169. DOI : http://dx.doi .org/10.1145/2901790.2901828

[44] Christian Weichel, Jason Alexander, Abhijit Karnik, and Hans Gellersen. 2015a. SPATA: Spatio-Tangible Tools for Fabrication-Aware Design. In Proceedings of the Ninth International Conference on Tangible, Embedded, and Embodied Interaction (TEI '15). ACM, New York, NY, USA, 189-196. DOI :

http://dx.doi.org/10.1145/2677199.2680576

[45] Christian Weichel, John Hardy, Jason Alexander, and Hans Gellersen. 2015b. ReForm: Integrating Physical and Digital Design Through Bidirectional Fabrication. In Proceedings of the 28th Annual ACM Symposium on User Interface Software \& Technology (UIST'15). ACM, New York, NY, USA, 93-102. DOI : http://dx.doi.org/10.1145/2807442.2807451
[46] Christian Weichel, Manfred Lau, David Kim, Nicolas Villar, and Hans W. Gellersen. 2014. MixFab: A Mixed-reality Environment for Personal Fabrication. In Proceedings of the SIGCHI Conference on Human Factors in Computing Systems (CHI'14). ACM, New York, NY, USA, 3855-3864. DOI : http://dx.doi.org/10.1145/2556288.2557090

[47] Karl D.D. Willis, Juncong Lin, Jun Mitani, and Takeo Igarashi. 2010. Spatial Sketch: Bridging Between Movement \& Fabrication. In Proceedings of the Fourth International Conference on Tangible, Embedded, and Embodied Interaction (TEI'10). ACM, New York, NY, USA, 5-12. DOI :

http://dx.doi.org/10.1145/1709886.1709890

[48] Karl D.D. Willis, Cheng Xu, Kuan-Ju Wu, Golan Levin, and Mark D. Gross. 2011. Interactive Fabrication: New Interfaces for Digital Fabrication. In Proceedings of the Fifth International Conference on Tangible, Embedded, and Embodied Interaction (TEI '11). ACM, New York, NY, USA, 69-72. DOI :

http://dx.doi.org/10.1145/1935701.1935716

[49] Junichi Yamaoka and Yasuaki Kakehi. 2013. dePENd: Augmented Handwriting System Using Ferromagnetism of a Ballpoint Pen. In Proceedings of the 26th Annual ACM Symposium on User Interface Software and Technology (UIST'13). ACM, New York, NY, USA, 203-210. DOI :

http://dx.doi.org/10.1145/2501988.2502017

[50] Mayu M. Yamashita, Junichi Yamaoka, and Yasuaki Kakehi. 2013. Enchanted Scissors: A Scissor Interface for Support in Cutting and Interactive Fabrication. In ACM SIGGRAPH 2013 Posters (SIGGRAPH'13). ACM, New York, NY, USA, 33:1-33:1. DOI : http://dx.doi.org/10.1145/2503385.2503422

[51] Amit Zoran and Joseph A. Paradiso. 2013. FreeD: A Freehand Digital Sculpting Tool. In Proceedings of the SIGCHI Conference on Human Factors in Computing Systems (CHI'13). ACM, New York, NY, USA, 2613-2616. DOI :

http://dx.doi.org/10.1145/2470654.2481361

[52] A. Zoran, R. Shilkrot, P. Goyal, P. Maes, and J. A. Paradiso. 2014. The Wise Chisel: The Rise of the Smart Handheld Tool. IEEE Pervasive Computing 13, 3 (July 2014), 48-57. DOI :

http://dx.doi.org/10.1109/MPRV. 2014.59 